\title{
A novel blood test for tuberculosis prevention and treatment
}

Almost 1 in every 100 South Africans is diagnosed with active tuberculosis (TB) disease every year, an incidence that ranks among the highest of the world's 22 high-TB-burden countries; TB accounted for an astounding $14.6 \%$ of deaths among 15 - 44-year-olds in South Africa (SA) in 2014. ${ }^{[1]}$ Globally, TB is recognised as the leading cause of mortality by an infectious agent, with 1.4 million deaths and 10.4 million new TB cases in 2015. ${ }^{[2]}$ Although most forms of TB are treatable, prompt diagnosis is challenging and passive case-finding approaches have failed to control the epidemic. The estimated $80 \%$ of SA adults who are latently infected with Mycobacterium tuberculosis form a massive reservoir for future reactivation cases. ${ }^{[3,4]}$ Indeed, even if all new M. tuberculosis infections were prevented, the incidence of TB stemming from the global reservoir of 1.7 billion latently infected people (23\% of the world population) would be 16.5 per 100000 person-years in 2035 , falling short of the 2050 target for eradicating TB. ${ }^{[4]}$ As preventive therapy at this scale is not feasible, no single current intervention is likely to achieve the goals of The End TB Strategy. ${ }^{[5]}$

There is an urgent need for earlier TB case identification, using novel non-sputum-based diagnostics, linked to more effective preventive and curative strategies. A blood-based triage test that allows targeted investigation of individuals with active and sub-clinical TB disease, including asymptomatic individuals at highest risk of progression from latency to disease, could help to shorten the time to treatment start, or even prevent incipient disease before symptoms emerge. ${ }^{[6,7]}$ The vision of a TB blood test to identify high-risk individuals for targeted preventive or curative therapy is within reach.

We recently identified a blood ribonucleic acid (RNA) signature capable of predicting TB disease before the onset of symptoms. ${ }^{[8]}$ This RNA signature, which measures expression of interferon response genes, has been translated into a polymerase chain reaction (PCR) test and was independently validated in cohorts from The Gambia and SA. The 16-gene PCR test predicted incident TB in adolescents, with a specificity of $82 \%$ and sensitivity of $70 \%$ within a year of testing - in the combined training and test sets. ${ }^{[8]}$ To improve throughput of the test, we have refined the RNA signature to 11 genes, resulting in prognostic specificity of $84 \%$ and sensitivity of $71 \%$ (area under the curve $=0.76) 1$ year before the onset of TB disease (A PennNicholson - unpublished data). The PCR assay has been qualified, and engagement is underway with commercial partners to translate this test technology to a smaller, simpler, faster and cheaper pointof-care test.

Prevention of TB disease arising from latent infection is a key requirement to achieving global elimination targets, but the inability to detect $M$. tuberculosis in vivo during latent infection constitutes a major hurdle to effective prognostics. ${ }^{[4]}$ The Foundation for Innovative New Diagnostics (FIND) has proposed that the target product profile of a prognostic test for incident TB should have at least $75 \%$ sensitivity and $75 \%$ specificity (and optimally $>90 \%$ for both) ${ }^{\left[{ }^{[9]}\right.}$ Neither the tuberculin skin test nor the interferon-gamma release assay (IGRA) meets this minimum standard. For example, if IGRA were used as a prognostic test in SA, the number needed to treat (NNT) to prevent one case of TB would be 85 , based on an assumed $2 \%$ cumulative incidence of TB over 2 years and $50 \%$ effectiveness of isoniazid preventive therapy (IPT). However, if the RNA signature performs as predicted, the NNT would be more than halved, which might make large-scale, targeted TB preventive therapy feasible, even in high-TB-incidence countries. ${ }^{[10,11]}$

The RNA signature is being assessed in a clinical trial to identify healthy adults at high risk of TB disease, and to test whether targeted short-course preventive therapy ( 3 months of once weekly, high-dose isoniazid plus rifapentine (3HP)) can prevent TB disease in individuals who test positive for the RNA signature. The Correlate of Risk (COR) Targeted Intervention Study (CORTIS), ${ }^{[12]}$ currently underway at 5 sites across SA, intends to screen $>10000$ healthy adults for the RNA signature and follow 3200 participants over 15 months for incident TB disease. CORTIS tests the hypotheses that high-risk signaturepositive individuals $(\mathrm{COR}+)$ will have a higher incidence of active $\mathrm{TB}$ disease than low-risk signature-negative individuals (COR-); and that $3 \mathrm{HP}$ will reduce the incidence of $\mathrm{TB}$ disease in $\mathrm{COR}+$ participants compared with $\mathrm{COR}+$ participants under active surveillance.

The RNA signature might also have diagnostic utility for undiagnosed, sub-clinical TB disease when deployed as a triage tool to trigger investigation in otherwise healthy, RNA signature-positive individuals. We have provisionally demonstrated $93 \%$ specificity and $92 \%$ sensitivity to discriminate healthy South Africans with latent $M$. tuberculosis infection from those with active TB disease (F Darboe et al. - unpublished data). Within CORTIS, we will further assess the use of the RNA signature to diagnose prevalent TB in HIV-negative adults. If successful, this would enable targeted investigation of RNA signature-positive individuals for sub-clinical TB disease, allowing early curative treatment; RNA signature-positive individuals found not to have active disease would be offered preventive therapy; and those found to be RNA signature negative would be spared unnecessary intervention (Fig. 1).

It is estimated that $11 \%$ of South Africans live with HIV; yet, $57 \%$ of the burden of all TB in SA is borne by those living with HIV. ${ }^{[2]} \mathrm{HIV}$ infection significantly elevates the risk of TB disease. ${ }^{[13]}$ Performance of the RNA signature will also be tested in an observational study of SA adults living with HIV, in parallel with CORTIS. If the prognostic RNA signature performs as expected, it might also be used to identify HIV-positive individuals at highest risk of TB disease within a year of testing, and thus trigger initiation of targeted, short-course preventive therapy regimens that may in future replace chronic IPT. ${ }^{[14]}$

Should the CORTIS screen-and-treat strategy prove efficacious in predicting and reducing the incidence of TB disease by targeted preventive therapy, the critical question is whether implementation would be feasible for the SA healthcare system. Key considerations are: (i) test performance; (ii) population level impact; (iii) cost/benefit ratio; (iv) operational feasibility; and ( $v$ ) political commitment. RNA signature performance is being tested prospectively in CORTIS, but preliminary models predict that a TB screen-and-treat strategy that reached $30 \%$ of HIV-negative SA adults annually could reduce the

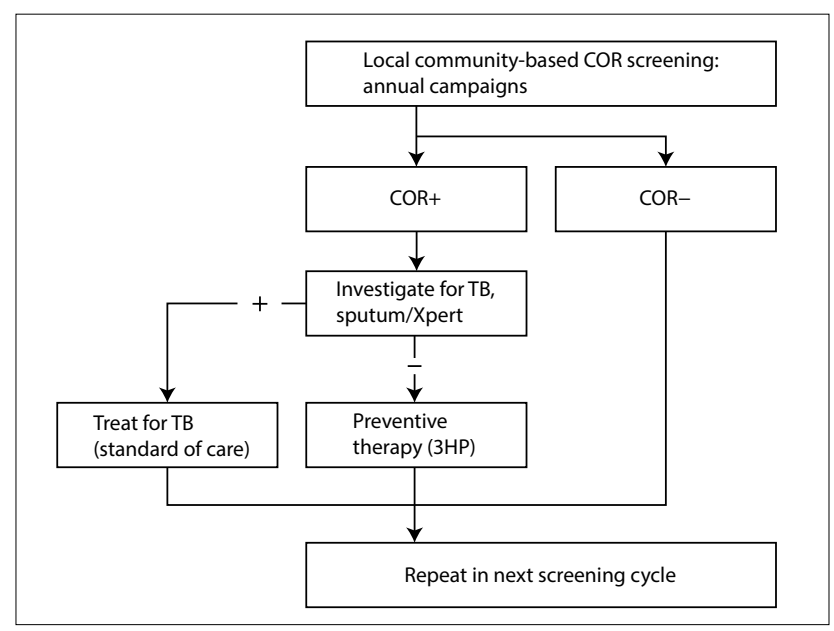

Fig. 1. Proposed RNA signature screen-and-treat algorithm. 
national TB incidence by $14 \%$ (95\% confidence interval (CI) $11-18 \%$ ) over 5 years. If extended to both HIV-negative and HIV-positive people, estimations suggest a reduction in TB incidence of $29 \%$ (95\% CI $24-32 \%$ ), and in TB mortality of 35\% (95\% CI $29-37 \%$ ), within 5 years (R G Sumner and T White - unpublished data). In the face of the potential impact, the cost/benefit assessment of such a strategy needs to be compared with that of untargeted IPT for all latently infected South Africans, which is clearly not feasible. SA already has an established health infrastructure for large-scale HIV test-and-treat programmes, which could be augmented to enable annual community-based screening for TB, using an affordable nearpoint-of-care device. Finally, with nearly 100000 South Africans dying from TB in $2015{ }^{[2]}$ scientific, pharmaceutical and governmental stakeholders have a collective responsibility to act promptly on new $\mathrm{TB}$ research findings and implement novel strategies to save lives. The current inadequate tools for screening, diagnosing, treating and preventing TB must be urgently and significantly improved if we are to end TB in our lifetime.
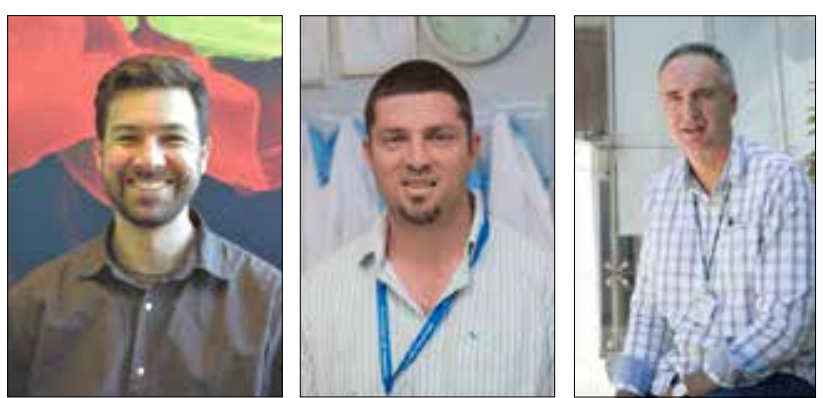

Adam Penn-Nicholson, Thomas J Scriba, Mark Hatherill South African Tuberculosis Vaccine Initiative, Institute of Infectious Disease and Molecular Medicine, and Division of Immunology, Department of Pathology, Faculty of Health Sciences, University of Cape Town, South Africa adam.penn-nicholson@uct.ac.za
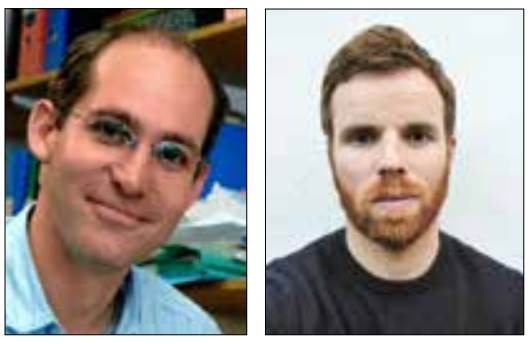

Richard G White, Tom Sumner

TB Modelling Group, TB Centre, Faculty of Epidemiology and Public Health, London School of Hygiene and Tropical Medicine, London, UK

1. Statistics South Africa. Mortality and Causes of Death in South Africa, 2014: Findings from Death Notification. Pretoria: Statistics SA, 2015.

Writhealth Organization. Global Tuberculosis Report 2016:1-211. Geneva: WHO, 2016. Mahomed H, Hughes EJ, Hawkridge T, et al. Comparison of mantoux skin test with three generations
of a whole blood IFN-gamma assay for tuberculosis infection. Int J Tuberc Lung Dis 2006:10(3):310316. http://dx.doi.org/10.1164/rccm.200608-10880C

4. Houben RMGJ, Dodd PJ. The global burden of latent tuberculosis infection: A re-estimation using mathematical modelling. PLoS Med 2016;13(10):e1002152. http://dx.doi.org/10.1371/journal. pmed. 1002152

5. Houben RMGJ, Menzies NA, Sumner T, et al. Feasibility of achieving the 2025 WHO global tuberculosis targets in South Africa, China, and India: A combined analysis of 11 mathematical models. Lancet Glob Health 2016;4(11):e806-815. http://dx.doi.org/10.1016/S2214-109X(16)30199-1 Malherbe ST, Shenai S, Ronacher K, et al. Persisting positron emission tomography lesion activity and Mycobacterium tuberculosis mRNA after tuberculosis cure. Nat Med 2016;22(10):1094-1100. http://

. Esmail H, Lai RP, Lesosky M, et al. Characterization of progressive HIV-associated tuberculosis using 2-deoxy-2-[(18)F]fluoro-D-glucose positron emission and computed tomography. Nat Med Zak DE Penn-Nicholson A Scriba TH et ab. A blood RNA

A, Scriba TJ, et al. A blood RNA signature for tuberculosis disease risk: A prospective cohort study. Lancet 2016;387(10035):2312-2322. http://dx.doi.org/10.1016/S0140-

. Foundation for Innovative New Diagnostics. Draft target product profile: Test for incipient 2_20OCT2016 2016. http://www.finddx.org/wp-cor

10. Petruccioli E, Scriba TJ, Petrone L, et al. Correlates of tuberculosis risk: Predictive biomarkers for progression to active tuberculosis. Eur Respir J 2016;pii:ERJ-01012-2016. http://dx.doi.org/ 10.1183/13993003.01012-2016

1. Zak D, Scriba TJ, Hatherill M, Penn-Nicholson A, Hanekom W. Predicting tuberculosis risk. Lancet 2016;388(10057):2233-2234. http:///x.doi.org/10.1016/S0140-6736(16)31653-1

2. The Correlate of Risk (COR) Targeted Intervention Study. http://www.clinicaltrials.gov.NCT02735590 (accessed 30 November 2016).

3. Lawn SD, Churchyard G. Epidemiology of HIV-associated tuberculosis. Curr Opin HIV AIDS 2009;4(4):325-333. http://dx.doi.org/10.1097/COH.0b013e32832c7d61

. WHO, 2015.

S Afr Med J 2017;107(1):4-5. DOI:10.7196/SAMJ.2017.v107i1.12230 